\title{
Association Among Self-Injury, Suicidal Tendency, and Personality and Psychological Characteristics in Korean Adolescents
}

\author{
Kyojin Yang ${ }^{1}$, Si Young Kim², Hyejin Kim³ , and Jun-Won Hwang ${ }^{1 凶}$ \\ ${ }^{1}$ Department of Psychiatry, Kangwon National University Hospital, Kangwon University School of Medicine, Chuncheon, Republic of Korea \\ ${ }^{2}$ OKmind Psychiatry Clinic, Seoul, Republic of Korea \\ ${ }^{3}$ Gangneung Ana-Hospital, Gangneung, Republic of Korea
}

\begin{abstract}
Objective To compare mental health problems and personality characteristics between adolescents with self-injury (SI) and suicidal tendency (ST), including suicidal ideation and suicide planning.

Methods The participants completed the Adolescent Personality and Mental Health Problems Screening Questionnaire, Third version (AMPQ-III), and Korean version of the Strengths and Difficulties Questionnaire (SDQ-Kr), and gave their demographic information. Based on the answered SI and ST items in AMPQ-III, the participants were divided into groups: without SI and ST, with SI and without ST, without SI and with ST, and with SI and ST. Analysis of variance (ANOVA) and post-hoc Scheffe test were used to determine group differences, stepwise multiple linear regression, and factors associated with SI and ST.

Results ANOVA yielded results for all categories of mental health problems. Significant differences were observed between the four groups. Conversely, for personality characteristics, only the sense of community item between the group without SI and with ST and the group without SI and ST were significantly different; sense of community was associated with ST $(\beta=-0.054, p=0.005)$. Among the mental health subscales, SI was significantly associated with conduct problems $(\beta=0.211, \mathrm{p}<0.001)$, emotional problems $(\beta=0.173$, $\mathrm{p}<0.001)$, peer problems $(\beta=0.085, p<0.001)$, and prosocial behavior $(\beta=0.073, p<0.001)$. ST was significantly associated with emotional symptoms $(\beta=0.317, \mathrm{p}<0.001)$, peer problems $(\beta=0.130, \mathrm{p}<0.001)$, conduct problems $(\beta=0.106, \mathrm{p}<0.001)$, hyperactivity $(\beta=0.050, \mathrm{p}=0.010)$, and prosocial behavior $(\beta=0.062, \mathrm{p}<0.001)$.

Conclusion The findings highlight the necessity of assessing psychological strength, difficulties, and personality characteristics for the prevention and early intervention of adolescent SI.

Psychiatry Investig 2022;19(1):37-43
\end{abstract}

Keywords Self-injury; Suicidal tendency; Psychopathology; Personality.

\section{INTRODUCTION}

Suicide and self-injury are considered serious social problems among the Korean youth of today. Intentional self-injury (suicide) from 2007 to 2019 accounts for the most common cause of death among adolescents. Moreover, the annual suicide rate among youths aged 9-24 was 7.7 per 100,000 in 2017. ${ }^{1}$ The most recent Korea Youth Risk Behavior Web-Based Survey to date found that approximately $13.1 \%$ of students exhibit-

Received: May 31, 2021 Revised: September 10, 2021

Accepted: October 9, 2021

$\triangle$ Correspondence: Jun-Won Hwang, MD, PhD

Department of Psychiatry, Kangwon National University Hospital, 156 Baengnyeong-ro, Chuncheon 24289, Republic of Korea

Tel: +82-33-258-2201, Fax: +82-33-258-2348, E-mail: huangjw@hanmail.net

(c) This is an Open Access article distributed under the terms of the Creative Commons Attribution Non-Commercial License (https://creativecommons.org/licenses/by$\mathrm{nc} / 4.0$ ) which permits unrestricted non-commercial use, distribution, and reproduction in any medium, provided the original work is properly cited. ed suicidal ideation in the last 12 months, and that girls (17.1\%) had a higher risk of suicide than boys $(9.4 \%){ }^{2}$ In general, suicide involves the following processes: suicidal ideation (i.e., a serious consideration to commit suicide), suicidal planning (i.e., creation of specific method for suicide), and suicide attempt (i.e., fatal behaviors for the purpose of killing oneself). It is carried out through a series of processes that lead to the completion of suicide (i.e., death as the final outcome of the suicide process), which is commonly used to describe these processes. ${ }^{3,4}$

Self-injury, which has been common in clinical settings, has been regarded as an imminent sign of suicide, especially in adolescents. ${ }^{5}$ However, in the recently revised Diagnosis and Statistics of Mental Disorders (DSM-5), it is defined as Non-suicidal self-injury (NSSI), characterized as not intended for death but for intentional self-inflicted damage to the surface of the body (e.g. cutting, burning, stabbing, hitting, excessive rubbing). NSSI was introduced as a diagnostic status item requir- 
ing further study. Self-injury without suicidal tendency is often aimed at gaining relief from negative emotions or cognitive states and solving an interpersonal difficulty, inducing a positive mood state. ${ }^{6}$ Some researchers have also suggested that selfinjury without suicidal intentions is done to escape from the feeling of being insensitive and empty, or for self-punishment. ${ }^{7}$

In a previous international study conducted with a community sample of adolescents, the prevalence of self-injury without suicidality was measured to be $5 \%-37 \%{ }^{4,8,9}$ It has been reported mainly in females. ${ }^{10-12}$ In a two-year reanalysis of the $s$ (AMPQ-III) results, which the Ministry of Education uses to annually survey all 1st grade middle and high school students nationwide, $37,373(8.3 \%)$ of the 450,1151 st grade middle school students and 30,625 (5.9\%) out of the 517,294 1st grade high school students who took in the test in 2017, and 40,505 (7.9\%) out of the 514,710 1st grade middle school students and 29,026 (6.4\%) out of the 452,107 high school students who took in the test in 2018 responded to "I have hurt myself intentionally." ${ }^{13}$

The results of several previous major studies examining the relationship between psychopathology and personality characteristics of adolescents with suicidality and self-injury are as follows. In a study using the Toronto Alexithymia Scale and Children's Depression Inventory to analyze 784 adolescents aged 13-15 years, alexithymia, depression, and peer relations significantly predicted self-injury. ${ }^{14}$ In a study with 717 middle school students using tools, such as Hospital Anxiety and Depression (HAD), Self-esteem scale, and the Korean children and Youth Panel Survey (KCYPS), alcohol use, anxiety, and parental abuse, were significantly associated with NSSI. ${ }^{15}$ In a study using the Korean version of the Child Behavior Checklist (KCBCL) to analyze 1,857 adolescents, factors, such as somatic problems, thought problems, delinquent behavior, and aggressive behavior, were independently associated with adolescent self-injury and suicidal ideation. ${ }^{16}$ In a study on the relationship between the severity of NSSI and the personality traits of the Big 5 Theory among 2,170 high school students in Taiwan, the mild self-injury group reported higher levels of neuroticism and openness than non-injurers. Conversely, in the severe selfinjury group, studies have reported higher levels of neuroticism, openness, as well as avoidance or emotional-focused coping, virtual social support, and lower levels of self-esteem. ${ }^{17}$

The 'Strength and Difficulties Questionnaire' (SDQ) is used to evaluate psychopathology both in internalising and externalising dimensions. In Hungary, the frequency of NSSI and the SDQ-internalising and externalising (SDQ-subthreshold) symptoms, there is a study that reported that there was a significant difference with the frequency of NSSI in the four groups. ${ }^{18} \mathrm{Neu}-$ rodevelopmental vulnerability in adolescence, especially during puberty, increases the relevance of emotional disorders and risk-taking behaviors. ${ }^{15}$ According to previous studies, depressive personality characteristics and trait anxiety are strong predictors of suicide attempts in adolescents. ${ }^{19}$

However, no research to date has examined the relationship between psychological and personality characteristics simultaneously to determine which of them predicts self-injury or suicidal tendency and how their interactions are. Therefore, this study aimed to determine the relationship between psychological and personality characteristics and self-injury and suicide tendency in Korean adolescents, and to identify related factors that influence the connection to psychiatric treatment.

\section{METHODS}

\section{Participants}

The data of this study were obtained during the standardization study of AMPQ- III. An online survey was conducted with 7,062 student respondents (3,770 1st grade middle school students and 3,292 1st grade high school students) enrolled in nine middle and high schools nationwide through a separate website from July 5 to 31,2016 . Of the 7,062 subjects, 3,172 $(44.9 \%)$ with written consent participated in this study. The study participants included 603 male and 681 female students in the first grade of middle school, and 898 male and 990 female students in the first grade of high school. This study was approved by the Institutional Review Board of the Chuncheon National Hospital (IRB No. 20160303-1). Informed consent was obtained from the caretakers of, and assent was obtained from all of the participants.

\section{Assessment and measures}

\section{Adolescent Personality and Mental Health Problems Screening Questionnaire, Third version (AMPQ- III)}

The AMPQ-III is a screening test for middle and high school students to evaluate their personality characteristics and mental health problems over the last three months via self-reporting. The AMPQ-III consists of 62 items in two major domains: Personality domain and Mental Health Problems domain. Most items, except those regarding perceived physical or mental health status and willingness to be referred, were scored using a four-point Likert scale from $0-3$. The test-retest reliability correlation coefficient was 0.92 .

The Personality domain was devised to evaluate a total of 6 personality characteristics, such as three internal personality characteristics (e.g., Self-efficacy, Openness, Sincerity), and three external personality characteristics (e.g., Interpersonal Understanding, Proactivity, and Sense of Community) of an individual, based on the Big 5 theory. Internal personality characteristics refer to the inner factors necessary to lead one's life 
better, and external personality characteristic reflects the necessary factors for smoothly leading relationships with others and the community group environment. Cronbach's $\alpha$ for the internal and external personality characteristics was 0.89 . Four factors for the Mental Health Problems domain in the AMPQIII were proposed: Psychological Distress, Mood Problems, Anxiety Problems, and Self-control Difficulties. In addition, 2 factors including bullying and suicide were added in order to screen students in crisis. ${ }^{13}$ Cronbach's $\alpha$ of the Mental Health Problems domain was 0.92 .

\section{Korean version of Strengths and Difficulties Questionnaire (SDQ-Kr)}

SDQ-Kr is a self-reporting test for children and adolescents in the community. It consists of 25 questions and uses a Likert scale of 0 points for "all," 1 point for "somewhat," and 2 points for "obviously" based on one's experience over the past six months. ${ }^{20}$ The scale score is calculated considering five factors: Prosocial Behavior, Hyperactivity, Emotional Symptoms, Conduct Problems, and Peer Problems. Among them, the total Prosocial Behavior score constitutes the total score for Strength, and the sum of the Hyperactivity, Emotional Symptoms, Behavior Problems, and Peer Problems scores constitutes the total Difficulty score. The SDQ-Kr scale Cronbach's $\alpha$ was reported as $0.50-$ $0.80 .^{21}$

\section{Statistical analysis}

The item about self-injury in AMPQ-III is "I have hurt myself intentionally." It is one question, and if there was one point and more in the item, it was considered to be an affirmative answer. In addition, the questions related to suicidal tendencies were: 1) "I felt like dying.", and 2) "I devised a detailed plan to kill myself at least once." If the sum of the two questions was one point and more, it was considered to be affirmative for suicidal tendency. Through this process, the participants were classified into four groups: 1) without self-injury and suicidal tendency, 2) with self-injury and without suicidal tendency, 3) without self-injury and suicidal tendency, and 4) with self-injury and suicidal tendency.

Descriptive statistics were performed on the ratio of these four clinical group, such as sex and grade, to indicate the characteristics of the groups. One-way analysis of variance (ANOVA) with post-hoc Scheffe test was used for comparison between the groups. Pearson's correlation analysis was performed to analyze the correlation between the scale scores that resulted in significant results in the comparison between groups and self-injury and suicidal tendency, and sex and age were adjusted. The dependent variables were self-injury and suicidal tendency, and the independent variable was a stepwise multiple linear analysis with a scale score that resulted in significant results between groups. In all analyses, statistical significance was set at $\mathrm{p}<0.05$. All statistical analyses were performed using IBM SPSS for Windows (version 23.0; IBM Corp., Armonk, NY, USA).

\section{RESULTS}

Demographic and clinical characteristics of the patients are presented in Table 1. The participants of this study consisted of 603 males and 681 females in the 1st grade of middle school, and 898 males and 990 females in the 1st grade of high school. Each was classified according to the result of the AMPQ-III self-injury-related question as follows: Without self-injury and suicidal tendency group, 2,526 (79.6\%); with self-injury and without suicidal tendency group, 93 (2.9\%); without self-injury and with suicidal tendency group, 384 (12.1\%); and with selfinjury and suicidal tendency group, 169 (5.3\%) (Table 1).

There were significant differences between the groups regarding all SDQ-Kr items. In the post-hoc Scheffe test, in the case of the score of the Prosocial Behavior subscale, which is classified as a total Strength score, the group without self-injury and with suicidal tendency scored lower than the group without self-injury and suicidal tendency. In the case of the Hyperactivity subscale, three groups with either self-injury or suicidal tendency had significantly higher scores than the group without self-injury and suicidal tendency. In the Emotional Symptom subscale, higher score was found in order of the group with self-injury and suicidal tendency, the group without selfinjury and with suicidal tendency, the group with self-injury and without suicidal tendency, and the group without self-injury and suicidal tendency. In contrast, in the case of the Conduct

Table 1. Demographic and clinical characteristics of the participants

\begin{tabular}{lc}
\hline \multicolumn{1}{c}{ Characteristics } & Value \\
\hline Total subjects, N & $3,172(100.0)$ \\
Grade & \\
$\quad$ Middle school 1st grade & $603(47.0)$ \\
$\quad$ Male & $681(53.0)$ \\
$\quad$ Female & \\
High school 1st grade & $898(47.6)$ \\
$\quad$ Male & $990(52.4)$ \\
$\quad$ Female & \\
Group & $2,526(79.6)$ \\
Without SI and ST & $93(2.9)$ \\
With SI and without ST (group 2) & $384(12.1)$ \\
Without SI and with ST SD (group 3) & $169(5.3)$ \\
With both SI and ST & \\
\hline Values are presented as N (\%). SI, self-injury; ST, suicidal tendency
\end{tabular}


Problem subscale, the groups ordered according to decreasing scores were: with suicidal ideation and suicidal tendency, with self-injury and without suicidal tendency, without selfinjury and with suicidal tendency, and without self-injury and suicidal tendency. In the case of the total Difficulty score consisting of the Peer Problem scale and the sum of four scales, namely, Hyperactivity, Emotional Symptoms, Conduct Problems, and Peer Problems, the scores groups ordered according to decreasing scores were: with suicidal ideation and suicidal tendency, without self-injury and with suicidal tendency, with self-injury and without suicidal tendency, and without self-injury and suicidal tendency (Table 2).

As a result of comparison between the four groups and six subscales of the Personality domain of AMPQ-III, there was a significant difference in the Sense of Community subscale. In the post-hoc Scheffe test, the group without self-injury and suicidal tendency showed significantly higher scores than the group without self-injury and with suicidal tendency (Table 3).

Table 4 shows the results of the stepwise multiple linear regression analysis of the effects of psychological characteristics and personality characteristics in adolescents on self-injury and suicidal tendencies. Self-injury showed significant results with Prosocial Behavior $(\beta=0.073, \mathrm{p}<0.001)$, Emotional Symptoms $(\beta=0.173, p<0.001)$, Conduct Problems $(\beta=0.211, p<$ $0.001)$, and Peer Problems $(\beta=0.085, \mathrm{p}<0.001)$. All variables showed $12 \%$ explanatory power. In the case of suicidal tendency, Prosocial Behavior $(\beta=0.062, \mathrm{p}<0.001)$, Hyperactivity $(\beta=0.050, p=0.010)$, Emotional Symptoms $(\beta=0.317, p<0.001)$, Conduct Problems $(\beta=0.106, \mathrm{p}<0.001)$, Peer Problems $(\beta=0.130$, $\mathrm{p}<0.001)$, and Sense of Community $(\beta=-0.054, \mathrm{p}<0.001)$ showed significant association, and all variables showed $22 \%$ explanatory power.

\section{DISCUSSION}

In this study, the psychological characteristics of the SDQ-Kr and personality characteristics in the Personality domain of the AMPQ-III were investigated in 3,172 adolescents, according to the self-injury and suicide tendency of the AMPQ-III. The results of this study are the first to investigate psychopathology and personality in four groups according to self-injury and suicidal tendencies and are partially consistent with the

Table 2. Comparison of SDQ-Kr variables according to self-injury or suicidal tendency

\begin{tabular}{|c|c|c|c|c|c|c|c|}
\hline \multirow[t]{2}{*}{ SDQ-Kr subscale } & $\begin{array}{l}\text { Without } \\
\text { SI and ST } \\
(\mathrm{N}=2,526)^{\mathrm{a}}\end{array}$ & $\begin{array}{c}\text { With SI } \\
\text { and without ST } \\
\quad(\mathrm{N}=93)^{\mathrm{b}} \\
\end{array}$ & $\begin{array}{l}\text { Without SI } \\
\text { and with ST } \\
(\mathrm{N}=384)^{\mathrm{c}}\end{array}$ & $\begin{array}{l}\text { With SI } \\
\text { and ST } \\
(\mathrm{N}=169)^{\mathrm{d}}\end{array}$ & \multirow[t]{2}{*}{$\mathrm{F}$} & \multirow[t]{2}{*}{$\mathrm{p}$} & \multirow[t]{2}{*}{ Post-hoc Scheffe test } \\
\hline & Mean \pm SD & Mean \pm SD & Mean \pm SD & Mean \pm SD & & & \\
\hline Prosocial behavior & $6.73 \pm 1.99$ & $6.77 \pm 2.02$ & $6.36 \pm 1.99$ & $6.54 \pm 2.11$ & 4.09 & 0.007 & $\mathrm{c}<\mathrm{a}$ \\
\hline Hyperactivity & $2.49 \pm 1.70$ & $3.35 \pm 1.69$ & $3.78 \pm 1.92$ & $3.77 \pm 1.81$ & 86.92 & $<0.001$ & $\mathrm{a}<\mathrm{b}, \mathrm{c}, \mathrm{d}$ \\
\hline Emotional symptoms & $2.27 \pm 1.86$ & $3.67 \pm 2.16$ & $4.53 \pm 2.25$ & $5.26 \pm 2.58$ & 254.38 & $<0.001$ & $\mathrm{a}<\mathrm{b}<\mathrm{c}<\mathrm{d}$ \\
\hline Conduct problems & $1.87 \pm 1.23$ & $2.90 \pm 1.69$ & $2.57 \pm 1.32$ & $3.37 \pm 1.64$ & 110.16 & $<0.001$ & $\mathrm{a}<\mathrm{c}<\mathrm{b}<\mathrm{d}$ \\
\hline Peer problems & $1.38 \pm 1.29$ & $2.01 \pm 1.67$ & $2.33 \pm 1.84$ & $2.66 \pm 1.65$ & 89.61 & $<0.001$ & $\mathrm{a}<\mathrm{b}, \mathrm{c}<\mathrm{d}$ \\
\hline Total score of strength & $6.73 \pm 1.99$ & $6.77 \pm 2.02$ & $6.36 \pm 1.99$ & $6.54 \pm 2.11$ & 4.093 & 0.007 & $\mathrm{c}<\mathrm{a}$ \\
\hline Total score of difficulties & $7.26 \pm 4.01$ & $11.29 \pm 5.02$ & $12.26 \pm 4.82$ & $14.41 \pm 5.39$ & 295.88 & $<0.001$ & $\mathrm{a}<\mathrm{b}, \mathrm{c}<\mathrm{d}$ \\
\hline
\end{tabular}

SDQ-Kr, Korean version of the Strengths and Difficulties Questionnaire; SI, self injury; ST, suicidal tendency; SD, standard deviation

Table 3. Comparison of Personality domain of AMPQ-III variables according to self-injury or suicidal tendency

\begin{tabular}{|c|c|c|c|c|c|c|c|}
\hline \multirow[t]{2}{*}{$\begin{array}{l}\text { Subscales in personality } \\
\text { domain of AMPQ-III }\end{array}$} & $\begin{array}{c}\text { Without } \\
\text { SI and ST } \\
(\mathrm{N}=2,526)^{\mathrm{a}}\end{array}$ & $\begin{array}{c}\text { With SI } \\
\text { and Without ST } \\
\quad(\mathrm{N}=93)^{\mathrm{b}}\end{array}$ & $\begin{array}{l}\text { Without SI } \\
\text { and with ST } \\
(\mathrm{N}=384)^{\mathrm{c}}\end{array}$ & $\begin{array}{l}\text { With SI } \\
\text { and ST } \\
(\mathrm{N}=169)^{\mathrm{d}}\end{array}$ & \multirow[t]{2}{*}{$\mathrm{F}$} & \multirow[t]{2}{*}{$\mathrm{p}$} & \multirow[t]{2}{*}{ Post-hoc Scheffe test } \\
\hline & Mean \pm SD & Mean \pm SD & Mean $\pm S D$ & Mean \pm SD & & & \\
\hline Sincerity & $7.44 \pm 2.52$ & $7.75 \pm 2.28$ & $7.09 \pm 2.51$ & $7.24 \pm 2.59$ & 2.93 & 0.032 & - \\
\hline Self-efficacy & $8.60 \pm 2.70$ & $8.52 \pm 2.64$ & $8.27 \pm 2.82$ & $8.55 \pm 2.82$ & 1.59 & 0.189 & - \\
\hline Openness & $7.88 \pm 2.52$ & $7.92 \pm 2.41$ & $7.69 \pm 2.58$ & $7.99 \pm 2.61$ & 0.77 & 0.512 & - \\
\hline Interpersonal understanding & $9.13 \pm 2.24$ & $9.46 \pm 1.90$ & $8.93 \pm 2.20$ & $8.92 \pm 2.23$ & 2.15 & 0.092 & - \\
\hline Sense of community & $9.39 \pm 2.31$ & $9.67 \pm 1.86$ & $8.98 \pm 2.48$ & $9.22 \pm 2.46$ & 4.08 & 0.007 & $\mathrm{c}<\mathrm{a}$ \\
\hline Proactivity & $7.18 \pm 2.80$ & $7.63 \pm 2.55$ & $6.93 \pm 2.77$ & $7.07 \pm 2.82$ & 1.88 & 0.131 & - \\
\hline
\end{tabular}

AMPQ-III, Adolescent Personality and Mental Health Problems Screening Questionnaire, Third version; SI, self-injury; ST, suicidal tendency; SD, standard deviation 
Table 4. Stepwise multiple regression of SDQ variable and personality characteristics on the self-injury and suicidal tendency (suicidal ideation/plan)

\begin{tabular}{|c|c|c|c|c|c|c|}
\hline & B & $\mathrm{SE}(\mathrm{B})$ & $\beta$ & $\mathrm{R}^{2}$ & $\mathrm{t}$ & $\mathrm{p}$ value \\
\hline Self-injury & & & & 0.120 & & \\
\hline (Constant) & -0.265 & 0.033 & & & -8.026 & $<0.001$ \\
\hline Conduct problems & 0.068 & 0.006 & 0.211 & & 11.394 & $<0.001$ \\
\hline Emotional symptoms & 0.035 & 0.004 & 0.173 & & 9.282 & $<0.001$ \\
\hline Peer problems & 0.025 & 0.085 & 0.085 & & 4.567 & $<0.001$ \\
\hline Prosocial behavior & 0.016 & 0.073 & 0.073 & & 4.118 & $<0.001$ \\
\hline Suicidal tendency & & & & 0.223 & & \\
\hline (Constant) & -0.311 & 0.082 & & & -3.804 & $<0.001$ \\
\hline Emotional symptoms & 0.114 & 0.006 & 0.317 & & 17.573 & $<0.001$ \\
\hline Peer problems & 0.07 & 0.010 & 0.13 & & 6.803 & $<0.001$ \\
\hline Conduct problems & 0.062 & 0.011 & 0.106 & & 5.797 & $<0.001$ \\
\hline Prosocial behavior & 0.024 & 0.007 & 0.062 & & 3.416 & 0.001 \\
\hline Hyperactivity & 0.022 & 0.007 & 0.05 & & 2.587 & 0.01 \\
\hline Sense of community & -0.018 & 0.008 & -0.054 & & -2.796 & 0.005 \\
\hline
\end{tabular}

SDQ, Strengths and Difficulties Questionnaire; B, regression coefficient; SE, standard error of B; $\beta$, standardized regression coefficient; $\mathrm{R}^{2}$, adjust

results of previous domestic and international studies. ${ }^{14,17,22,23}$ Of the participants, $262(8.3 \%)$ adolescents experienced selfinjury during the last three months, and 553 (17.4\%) adolescents had suicidal tendencies. In the previous study, the rate of self-injury experience was $6.9 \%$ in the UK, ${ }^{5}$ where the prevalence rate was surveyed in 41 schools over a period of one year through an anonymous self-report questionnaire. A study in China, which surveyed the prevalence rate from March to June 2008 through the Deliberate Self Harm screening question, found the rate to be $17.1 \% .{ }^{24}$ In a study in the United States, which used Composite International Diagnostic Interview to investigate lifetime suicidal behavior prevalence between 13-17 years of age, suicidal ideation, plans, and attempts were $12.1 \%$, $4.0 \%$, and $4.1 \%$, respectively. ${ }^{25}$

Regarding items in SDQ-Kr's Hyperactivity, Emotional Symptoms, Conduct Problems, and Peer Problem, three groups with either self-injury or suicidal tendency had significantly higher score than the group without self-injury and suicidal tendency. This is similar to a study by Bjärehed et al., ${ }^{22}$ who reported that self-injury without suicidal tendency had a significant correlation ( $r=0.22-0.32)$ with the SDQ subscales (Hyperactivity, Emotional Symptoms, Conduction Problem, and Peer Problem) in a study with 1,052 Swedish adolescents. In this study, the group without self-injury and with suicidal tendency scored significantly higher than the group with self-injury and without suicidal tendency in the Emotional Symptom subscale, which evaluates worries, fear, depressed mood, and somatic concerns. These results seem to be partially in line with the research findings that depressive mood in adolescents is internalized and can appear physically and predict suicidal behavior. ${ }^{26}$ Converse- ly, in the Conduct Problem subscale in this study, the group without self-injury and with suicidal tendency scored lower than the group with self-injury and without suicidal tendency. Liu et al. ${ }^{23}$ used the Child Behavior Checklist to measure behavioral problems after dividing 11,831 adolescents, with an average age of 14.97 years, in China, into four groups according to the presence or absence of self-injury and suicide attempts. To measure psychological vulnerability, the Beck Hopelessness Scale was used. As a result, delinquent behavior (suicide attempt only: $60.30 \pm 20.21$ vs. NSSI: $54.02 \pm 11.00$ ) and aggressive behavior (suicide attempt only: $59.43 \pm 16.20$ vs. NSSI: $55.27 \pm 11.08$ ) corresponded to externalizing in behavior problem, trait anger (suicide attempt only: $58.51 \pm 15.16$ vs. NSSI:

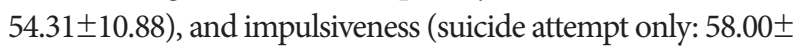
17.21 vs. NSSI: $54.29 \pm 10.88$ ) in psychological vulnerability. In terms of psychopathology, the group without self-injury and with suicidal tendency had a significantly higher $\mathrm{T}$ score than the group with self-injury and without suicidal tendency.

In this study, regarding the six subscales, namely, Sincerity, Self-efficacy, Openness, Interpersonal Understanding, Sense of Community, and Proactivity, under the Personality domain of AMPQ-III, the group without self-injury and with suicidal tendency showed a significantly lower score than the group without self-injury and suicidal tendency only in the Sense of Community subscale. This is consistent with the results of Hasking et al., ${ }^{27}$ which investigated the relationship between personality characteristics and self-injury as similar to this study, wherein none of the personality characteristics based on the Big 5 theory could predict self-injury alone.

In this study, the results of stepwise multiple linear regression 
were conducted to analyze the effects of psychological characteristics and personality characteristics on self-injury and suicidal tendency. In SDQ-Kr, Prosocial Behavior, Emotional Symptoms, Conduct Problems, and Peer Problems were significantly predicted for both self-injury and suicidal tendency. The four psychological characteristics that predict both self-jury and suicidal tendencies suggest that they are a common psychopathology between self-injury and suicidal tendency. ${ }^{23}$ In a previous study, ${ }^{28}$ prosocial behavior was reported as a factor that can predict suicide and self-injury; however, in this study, both self-injury and suicidal tendencies showed significantly positive effects, suggesting that at least self-injury or suicidal tendency in the participants of this study is prosocial on the one hand, but on the other hand, is a way to express the difficulties experienced in interpersonal relationships in adolescence. Another previous study has shown that childhood emotional dysregulation increases the risk of adult depression and suicide attempts. ${ }^{29}$ Conduct Problems are also high in adolescent depression and coexisting diseases, and past studies have reported associations with both self-injury and suicidal ideation and attempts. $^{30}$

Here, in addition to the four aforementioned psychological characteristics of suicidal tendency, Hyperactivity in SDQ-Kr and Sense of Community in the Personality domain of the AMPQ-III were included as predictive variables; attentiondeficit/hyperactivity disorder (ADHD), a representative disease characterized by hyperactivity, is a risk factor for suicidal accidents and suicide attempts in children and adolescents. ${ }^{31}$ Decision-making impairment and high risk-willingness that accompany ADHD may additionally increase the risk of suicide. ${ }^{32,33}$ In the case of Sense of Community, self-injury was not predicted, but it has a negative association with suicidal tendencies. This suggests that a sense of community can act as a protective factor for adolescents, and the introduction of activities and programs that can increase the sense of community in suicide prevention education for adolescents is required in the future.

Several limitations should be considered when interpreting our results. First, this study is a cross-sectional study consisting of data collection through a self-report questionnaire, and there are limitations in determining the causal relationship between factors. Second, there is a possibility that some subjects responded defensively because the contents of the questionnaire conducted to grasp the psychological characteristics in this study were often related to psychopathology. Third, the analysis of suicidal tendency in this study was limited to suicidal ideation and suicide planning, and therefore, did not include suicide attempts, as in previous studies. Also, in the clinical field and community, self-injury, suicidal ideation and plan were mixed, and there was a part of trying to solve suicidal ide- ation with self-injury. This part is described as suicidal tendency by combining suicidal ideation and suicide planning in this study. Fourth, due to the characteristics of the questionnaire study, clinical diagnosis information about the subject was not obtained, and there is a limitation in applying it to the clinical field.

In conclusion, early detection and intervention of self-injury and suicidal tendency in adolescence can reduce negative consequences during adolescence, as well as treat coexisting psychiatric disorders and correct coping with stress sources to reduce negative consequences leading to adulthood. The results of this study are intended to aid in understanding the characteristics of adolescents who have a tendency toward selfinjury and suicidal tendencies in clinical settings in the future, and to construct educational and intervention programs for students at risk of depression, self-injury, and suicide in the future. This is expected to provide useful information for implementation.

\section{Availability of Data and Material}

The datasets generated or analyzed during the study are available from the corresponding author on reasonable request.

\section{Conflicts of Interest}

The authors have no potential conflicts of interest to disclose.

\section{Author Contributions}

Conceptualization: Jun-Won Hwang, Kyojin Yang. Data curation: all authors. Formal analysis: Jun-Won Hwang, Si Young Kim, Kyojin Yang. Investigation: Jun-Won Hwang, Kyojin Yang. Methodology: Jun-Won Hwang, Kyojin Yang. Project Administration: all authors. Resources: all authors. Software: Jun-Won Hwang, Kyojin Yang. Supervision: Jun-Won Hwang. Validation: all authors. Visualization: Si Young Kim, Hyejin Kim, Kyojin Yang. Writing—original draft: all authors. Writing—review \& editing: all authors.

\section{ORCID iDs}

Kyojin Yang https://orcid.org/0000-0001-6152-3022

Si-Young Kim https://orcid.org/0000-0001-6425-1181

Hyejin Kim https://orcid.org/0000-0002-5446-0358

Jun-Won Hwang https://orcid.org/0000-0001-5407-8514

\section{Funding Statement \\ None}

\section{REFERENCES}

1. Ministry of Gender Equality and Family. 'Debate for the Prevention of Suicide and Self-Injury for Youth Search for solutions to the problem of suicide and self-harm for youth'. National Youth Policy 2nd Forum, Korea. Oct 28, 2019.

2. Kim HO. The effect of adolescent health behavior on the prevalence of allergic diseases based on the 15th (2019) Korea Youth Risk Behavior Web-Based Survey. J Korean Soc Sch Health 2020;33:125-138.

3. Australian Government Department of Health; 2009. Available at: https://www1.health.gov.au/internet/publications/publishing.nsf/Content/mental-pubs-m-mhaust2-toc $\sim$ mental-pubs-m-mhaust2hig mental-pubs-m-mhaust2-hig-sui. Accessed May 20, 2021.

4. Swannell SV, Martin GE, Page A, Hasking P, St John NJ. Prevalence of 
nonsuicidal self-injury in nonclinical samples: systematic review, metaanalysis and meta-regression. Suicide Life Threat Behav 2014;44:273303.

5. Hawton K, Rodham K, Evans E, Weatherall R. Deliberate self harm in adolescents: self report survey in schools in England. BMJ 2002;325: 1207-1211.

6. American Psychiatric Association. Diagnostic and Statistical Manual of Mental Disorders (5th Edition) Washington, D.C: American Psychiatric Publishing; 2013.

7. Lieb K, Zanarini MC, Schmahl C, Linehan MM, Bohus M. Borderline personality disorder. Lancet Psychiatry 2004;364:453-461.

8. Muehlenkamp JJ, Claes L, Havertape L, Plener PL. International prevalence of adolescent non-suicidal self-injury and deliberate self-harm. Child Adolesc Psychiatry Ment Health 2012;6:10.

9. Zetterqvist M, Lundh LG, Dahlström Ö, Svedin CG. Prevalence and function of non-suicidal self-injury (NSSI) in a community sample of adolescents, using suggested DSM-5 criteria for a potential NSSI disorder. J Abnorm Child Psychol 2013;41:759-773.

10. De Leo D, Heller TS. Who are the kids who self-harm? An Australian self-report school survey. Med J Aust 2004;181:140-144.

11. Kann L, McManus T, Harris WA, Shanklin SL, Flint KH, Queen B, et al. Youth risk behavior surveillance-United States, 2017. MMWR CDC Surveill Summ 2018;67:1-114.

12. Claes L, Vandereycken W, Vertommen H. Self-injury in female versus male psychiatric patients: a comparison of characteristics, psychopathology and aggression regulation. Pers Individ Dif 2007;42:611-621.

13. Hwang JW, Kim DI, Bahn GH, Ahn DH, Lee YS, et al. Report of Development of Students' Emotional and Behavioral Problems. Gyeonggi-do, 2017.

14. Lee WK. Psychological characteristics of self-harming behavior in Korean adolescents. Asian J Psychiatr 2016;23:119-124.

15. Kim M, Yu J. Factors contributing to non-suicidal self injury in Korean adolescents. J Korean Acad Community Health Nurs 2017;28:271-279.

16. Shin YM, Chung YK, Lim KY, Lee YM, Oh EY, Cho SM. Childhood predictors of deliberate self-harm behavior and suicide ideation in Korean adolescents: a prospective population-based follow-up study. J Korean Med Sci 2009;24:215-222.

17. Lin MP, You J, Ren Y, Wu JY, Hu WH, Yen CF, et al. Prevalence of nonsuicidal self-injury and its risk and protective factors among adolescents in Taiwan. Psychiatry Res 2017;255:119-127.

18. Horváth LO, Győri D, Komáromy D, Mészáros G, Szentiványi D, Balázs J. Nonsuicidal self-injury and suicide: the role of life events in clinical and non-clinical populations of adolescents. Front Psychiatry 2020; 11:370.

19. Ohring R, Apter A, Ratzoni G, Weizman R, Tyano S, Plutchik R. State and trait anxiety in adolescent suicide attempters. J Am Acad Child Adolesc Psychiatry 1996;35:154-157.
20. Goodman R, Ford T, Simmons H, Gatward R, Meltzer H. Using the Strengths and Difficulties Questionnaire (SDQ) to screen for child psychiatric disorders in a community sample. Br J Psychiatry 2000; 177:534-539.

21. Ahn JS, Jun SK, Han JK, Noh KS, Goodman R. The development of a Korean version of the Strengths and Difficulties Questionnaire. J Korean Neuropsychiatr Assoc 2003;42:141-147.

22. Bjärehed J, Wångby-Lundh M, Lundh LG. Nonsuicidal self-injury in a community sample of adolescents: Subgroups, stability, and associations with psychological difficulties. J Res Adolesc 2012;22:678-693.

23. Liu ZZ, Chen H, Bo QG, Chen RH, Li FW, Lv L, et al. Psychological and behavioral characteristics of suicide attempts and non-suicidal selfinjury in Chinese adolescents. J Affect Disord 2018;226:287-293.

24. Wan YH, Hu CL, Hao JH, Sun Y, Tao FB. Deliberate self-harm behaviors in Chinese adolescents and young adults. Eur Child Adolesc Psychiatry 2011;20:517-525.

25. Nock MK, Green JG, Hwang I, McLaughlin KA, Sampson NA, Zaslavsky AM, et al. Prevalence, correlates, and treatment of lifetime suicidal behavior among adolescents: results from the National Comorbidity Survey Replication Adolescent Supplement. JAMA Psychiatry 2013;70:300-310.

26. Bettes BA, Walker E. Symptoms associated with suicidal behavior in childhood and adolescence. J Abnorm Child Psychol 1986;14:591-604.

27. Hasking PA, Coric SJ, Swannell S, Martin G, Thompson HK, Frost AD. Brief report: emotion regulation and coping as moderators in the relationship between personality and self-injury. J Adolesc 2010;33:767773.

28. Pettingell SL, Bearinger LH, Skay CL, Resnick MD, Potthoff SJ, Eichhorn J. Protecting urban American Indian young people from suicide. Am J Health Behav 2008;32:465-476.

29. Adrian M, Zeman J, Erdley C, Lisa L, Sim L. Emotional dysregulation and interpersonal difficulties as risk factors for nonsuicidal self-injury in adolescent girls. J Abnorm Child Psychol 2011;39:389-400.

30. Vander Stoep A, Adrian M, Mc Cauley E, Crowell SE, Stone A, Flynn C. Risk for suicidal ideation and suicide attempts associated with cooccurring depression and conduct problems in early adolescence. Suicide Life Threat Behav 2011;41:316-329.

31. Balazs J, Miklósi M, Keresztény Á, Dallos G, Gádoros J. Attention-deficit hyperactivity disorder and suicidality in a treatment naïve sample of children and adolescents. J Affect Disord 2014;152:282-287.

32. Miller M, Sheridan M, Cardoos SL, Hinshaw SP. Impaired decisionmaking as a young adult outcome of girls diagnosed with attentiondeficit/hyperactivity disorder in childhood. J Int Neuropsychol Soc 2013;19:110-114.

33. Humphreys KL, Lee SS. Risk taking and sensitivity to punishment in children with ADHD, ODD, ADHD+ ODD, and controls. J Psychopathol Behav Assess 2011;33:299-307. 